A NOTE ON THE TRANSIENT

GAS FLOW PROBIEM

By A. Y. Sakakura

Trace Flemertis Investigations Report 329

UNITFD STATES DEPARTMENT OF THE INTERIOR

GEOLOGICAL SURVEI 


\section{UNITED STATES - \\ DEPARTMENT OF THE INTERIOR \\ GEOLOGICAL SURVEY \\ WASHINGTON 25, D.C.}

JUL 161953

$A B C-1340 / 3$

Dr. T. H. Johnson, Director

Dirision of Research

U. S. Atomic Energy Comission

l6th Street \& Constitution Are., N.W.

Washingt on 25, D. C.

Dear Dr. Johnson:

Transmitted herewith is one copy of TEI-329, "A note on the transient gas flow problem," by A. Y. Sakakura, June 1953.

We plan to publish this report in either Geophysics or the Journal of Petroleum Technology. An acknowledgment to the Division of Research will be inserted into the text of the report before it is subnitted for publication.

Sincerely yours,

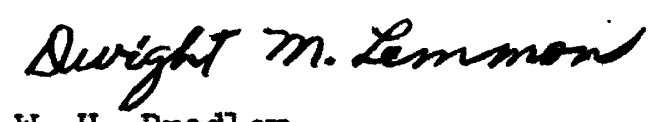

for w. H. Bradley

Chief Geologist

JAN 162001 
Physics

This document consists of 12 pages, including $i$ and ii. Series $A$

\section{UNITED STATES DEPARTMENT OF THE INTERIOR}

GEOLOGICAL SURVEY

A NOTE ON THE

TRANSIENT GAS FLOW PROBLEM*

By

A. Y. Sakakura

June 1953

Trace Elements Investigations Report 329

This preliminary report is distributed without editorial and technical review for conformity with official standards and nomenclature. It is not for public inspection or guotation.

Whis report concerns work done on behalf of the Division of Research of the U. S. Atomic Energy Comnission 
Distribution (Series A)

American Cyanamid Company, Winchester .

Argonne National Laboratory . . .

Atomic Energy Commission, Washington

Battelle Memorial Institute, Columbus

Carbide and Carbon Chemicals Company, Y-12 Area.

Division of Raw Materials, Butte.

Division of Raw Materials, Denver

Division of Raw Materials, Grants

Division of Raw Materials, Hot Springs.

Division of Raw Materials, New York.

Division of Raw Materials, Richfield

Division of Raw Materials, Salt Lake City.

Division of Raw Materials, Washington .

Division of Research, Washington

Dow Chemical Company, Pittsburg 0

Exploration Division, Grand Junction Operation

Grand Junction Operations Office. . .

Technical Information Service, Oak Ridge .

Tennessee Valley Authority, Wilson Dam.

\section{U. S. Geological Survey:}

Alaskan Geology Branch, Washington.

Fuels Branch, Washington.

Geochemistry and Petrology Branch, Washington

Geophysics Branch, Washington.

Mineral Deposits Branch, Washington.

E. H. Bailey, San Francisco.

J.R. Cooper, Denver

N. Mo Denson, Denver .

C. E. Dutton, Madison.

Henry Faul, Denver.

$R$, P. Fischer, Grand Junction

L. S. Gardner, Albuquerque .

C. B. Hunt, Plant City

M。 $R$ 。 Klepper, Washington

A.H. Koschmann, Denver .

R。A.Laurence, Knoxville

D. M. Lemmon, Washington.

J. D. Love, Laramie

L.R. Page, Denver 。.

R. J. Roberts. Salt Lake City

Q. D. Singewald, Beltsville.

J. F. Smith, Jr., Denver.

R.W. Swanson, Spokane

A. E. Weissenborn, Spokane .

W. P. Williams, Joplin

TEPCO, Washington:

Resource Compilation Section

Reports Processing Section .

(Including master)
No. of copies

1
1
2
1
1
1
1
1
1
6
1
1
3
1
1
1
1
6
1




\title{
A NOTE ON THE TRANSIENT GAS FLOW PROBLEM
}

By A. Y. Sakakura

\begin{abstract}
Expressions have been obtained for pressure distribution, velocity, volume flux, and total cumulative production of a gas well as a function of time after opening of a closed-down well. Static conditions prevail initially, and after opening, the well produces at constant well bottom pressure. The effect of the nonlinearity in Muskat's isothermal gas flow equation is shown to be negligible. The calculations can be applied to problems involving short-term transient flow of gases, such as in experiments with radioactive tracers.
\end{abstract}

\section{INTRODUCTION}

In the course of studies made by the U. S. Geological Survey on the transport of radon by natural gas through a reservoir to a gas well, it became necessary to re-examine the transient behavior of gas flow after the opening of a closed-down well. This problem was treated approximately by Muskat (1937), but our interests require more detailed knowledge of the periods less than an hour after opening.

\section{CALCULATIONS}

The phenomenological theory of gas flow through a porous medium was developed by Muskat (1937), and, in the case of isothermal flow of gas, the relevant equations are -

$$
\nabla^{2}\left(p^{2}\right)=\frac{2 \mu f}{k} \frac{\partial p}{\partial t}
$$


with the boundary conditions, in the case of radial flow, that

$$
\begin{array}{llll}
p=p_{e} \text { when } & r=b \text { and } & t>0 \\
p=p_{w} & \text { when } & r=a \text { and } & t>0 \\
p=p_{e} & \text { when } & t=0 \text { and } & a \leqslant r \leqslant b
\end{array}
$$

$$
\vec{v}=-\frac{k}{\mu} \nabla p
$$

where:

$$
\begin{aligned}
& p=\text { pressure in atmospheres } \\
& P_{e}=\text { reservoir pressure } \\
& P_{w}=\text { well pressure } \\
& a=\text { well radius } \\
& b=\text { effective reservoir radius } \\
& \mu=\text { viscosity in centipoises } \\
& f=\text { porosity } \\
& k=\text { permeability in darcies } \\
& \vec{v}=\text { velocity of gas in centimeters per second. }
\end{aligned}
$$

With the change of variable $\psi \equiv p_{e}^{2}-p^{2}$ equation (1) becomes

$$
\text { (3) } \nabla^{2} \psi=\frac{1}{k \sqrt{1-\psi / p_{e}^{2}}} \frac{\partial \psi}{\partial t} \text { where } \frac{1}{k}=\frac{\mu f}{k p_{e}}
$$

with the boundary conditions that

$$
\begin{aligned}
& \Psi=0 \quad \text { when } \quad a \leqslant r \leqslant b, t=0 \\
& \psi=0 \quad \text { when } \quad r=b, t>0 \\
& \psi=\Delta p^{2}=p_{e}^{2}-p_{w}^{2}, \quad r=a, t>0
\end{aligned}
$$

An examination of (3) reveals that it closely resembles the equation of heat conduction. As $\psi<P_{e}^{2}$ and as the strongest singularity of $\psi$ is a step function at $r=a, t=0$, it is evident that the nonlinearity introduces no new singularities in the equation of heat conduction. In fact, the only effect of nonlinearity will be a change in the "diffusivity". By utilizing the two extreme values of "diffusivity" the bounds of the actual solution can be found.

The linear problem (with the radical equal to 1) was solved by means of Laplace Transforms (Carslaw and Jaeger, 1950, p. 280) and by the use of 
Fourier-Bessel Series (Muskat. 1937, p. 632) and has a solution of the form:

(4) $\varphi_{0}+\sum_{n=0}^{\infty} U\left(\alpha_{n} r\right) e^{-k \alpha_{n}^{2} t}$

where $\psi_{0}$ is the steady state solution, and where $-U\left(\alpha_{n} r\right)^{\prime} s$ are certain combinations of Bessel functions which satisfy the boundary conditions. The $\alpha_{n}$ 's are the roots of the equation -

(5) $Y_{0}\left(\alpha_{n} a\right) J_{0}\left(\alpha_{n} b\right)-J_{0}\left(\alpha_{n} a\right) Y_{0}\left(\alpha_{n} b\right)=0$

where the $J_{0}$ 's and the $Y_{0}$ 's are the zero order Bessel functions of first and second kinds.

The above solution is useless for our purpose, as too many terms must be retained for small values of time when typical values of $b=500$ feet and $a=1 / 4$ foot are used. We therefore consider a problem more suitable for our purpose. All physical disturbances are propagated at a finite velocity, so that the external boandary of the reservoir has no effect on the solution until the disturbance arrives there. Thus we may consider the problem of the infinite reservoir up to the time when the disturbance reached the boundary.

The analogous heat conduction problem was treated thoroughly by Ritchie (1949) who modified Carslaw and Jaeger's general method of solution. We shall only briefly indicate this method. Taking the Laplace transform, with respect to $t$, of the gas flow equation (with the radical equal to 1 ) results in a subsidiary differential equation of Bessel's form and is satisfied by the function -

$$
\text { (6) } \frac{\Delta p^{2}}{z} \frac{k_{0}\left(z^{1 / 2} \varphi\right)}{k_{0}\left(z^{1 / 2}\right)}
$$

where: $\quad Z$ is the transform variable with respect to $\boldsymbol{\tau}$

$$
\begin{aligned}
& \tau=\frac{\kappa}{a^{2}} t \sim 2 \times 10^{3} \text { seconds } \\
& k_{0} \text { is a modified Bessel function of the second }
\end{aligned}
$$
kind, zero order

$$
\rho=\frac{r}{a}
$$

$\Psi(\boldsymbol{\tau})$ is obtained by applying the complex inversion integral to the above function:

$$
\psi=\frac{\Delta p^{2}}{2 \pi i} \int_{\gamma_{-i \infty}}^{\gamma_{+i \infty}} \frac{1}{z} \frac{K_{0}\left(z^{1 / 2} \rho\right)}{K_{0}\left(z^{1 / 2}\right)} e^{z \tau} d z
$$


Similarly, the other functions may be represented as:

(8)

$$
|\vec{v}|=\frac{\frac{-k}{2 \mu 2} \frac{\Delta p^{2}}{2 \pi i} \int_{\gamma-i \infty}^{\gamma+i \infty} \frac{1}{z^{1 / 2}} \frac{K_{1}\left(z^{1 / 2} \rho\right)}{K_{0}\left(z^{1 / 2}\right)} e^{z \tau} d z}{\left(p_{e}^{2}-\frac{\Delta p^{2}}{2 \pi i} \int_{\gamma-i \infty}^{\gamma+i \infty} \frac{1}{z} \frac{K_{0}\left(z^{1 / 2} \rho\right)}{K_{0}\left(z^{1 / 2}\right)} e^{z \tau} d z\right)^{1 / 2}}
$$

the volume production rate (per unit thickness) is:

$$
Q=-\frac{\pi k}{\mu} \frac{\Delta p^{2}}{p_{w}} \frac{1}{2 \pi i} \int_{\gamma-i \infty}^{\gamma_{+i \infty}} \frac{1}{z^{1 / 2}} \frac{K_{1}\left(z^{1 / 2}\right)}{K_{0}\left(z^{\gamma / 2}\right)} e^{z \tau} d z
$$

and the accumulated volume production (per unit thickness) is:

$$
\text { (10) } \int_{0}^{t} Q d t=\frac{-\pi a^{2}}{K} \frac{k}{\mu} \frac{\Delta p^{2}}{p_{w}} \frac{1}{2 \pi i} \int_{\gamma-i \infty}^{\gamma_{+} i \infty} \frac{1}{z^{3 / 2}} \frac{K_{1}\left(z^{1 / 2}\right)}{K_{0}\left(z^{1 / 2}\right)} e^{z \tau} d z
$$

where the $K_{i}$ 's are modified Bessel functions of the second kind, first order. It is to be noted that even when $\mathcal{\tau}$ is quite large, $t$ remains relatively small. Also, large values of $\boldsymbol{\tau}$ correspond to small values of $z$. Therefore, $K_{0}$ and $K_{1}$ may be replaced by their convergent series for small $z$ and the indicated division performed. We evaluate the Laplace contour integral by transforming to a positive circuit about the negative real axis of the $z$-plane. We find -

$$
\begin{aligned}
& \frac{1}{2 \pi i} \int_{\gamma-i \infty 0}^{\gamma+i \infty} \frac{1}{z} \frac{K_{0}\left(z^{1 / 2} \rho\right)}{K_{0}\left(z^{1 / 2}\right)} e^{z \tau} d z \sim \\
& \quad 1+2 \log \rho I_{1,-1}(\tau)+\frac{1}{4}\left\{2\left(\rho^{2}-1\right)(\log \rho-1) I_{1,0}(\tau)+\right. \\
& \left.\quad+4 \log \rho I_{2,0}(\tau)\right\}+\frac{1}{64}\left\{\left[2 \rho^{4}(\log \rho-3 / 2)-\right.\right. \\
& \left.\quad-8 \rho^{2}(\log \rho-2)-13+6 \log \rho\right] I_{1,1}(\tau)+\left[16 \rho^{2}(\log \rho-1)-\right. \\
& \left.\left.\quad-26 \log \rho+16] I_{2,1}(\tau)+32 \log \rho I_{3,1}(\tau)\right]\right\}+\frac{2}{2304} O\left(\frac{\rho^{6}}{\tau^{3}}\right)
\end{aligned}
$$


and

$$
\text { (12) } \begin{aligned}
\frac{\rho}{2 \pi i} & \int_{\gamma-i \infty}^{\gamma+i \infty} \frac{1}{z^{1 / 2}} \frac{K_{1}\left(z^{1 / 2} \rho\right)}{K_{0}\left(z^{1 / 2}\right)} e^{z \tau} d z \sim \\
& -2 I_{1,-1}(\tau)+\frac{1}{4}\left\{\left[-2 \rho^{2}(2 \log \rho-1)+2\right] I_{1,0}(\tau)-4 I_{2,0}(\tau)\right\}+ \\
& +\frac{1}{64}\left\{\left[4 \rho^{4}\left(\frac{5}{2}-2 \log \rho\right)+8 \rho^{2}(2 \log \rho-3)-6\right] I_{1,1}(\tau)+\right. \\
& \left.+\left[16 \rho^{2}(1-2 \log \rho)+26\right] I_{2,1}(\tau)-32 I_{3,1}(\tau)\right\}+ \\
& +\frac{6}{2304} 0\left(\frac{\rho^{6}}{\tau^{3}}\right)
\end{aligned}
$$

where $I_{k, \nu}=\frac{1}{2 \pi i} \int_{-\infty}^{(0+)} \frac{z^{\nu} e^{z \tau}}{\left[\log \frac{\sigma^{2} z}{4}\right]^{k}} d z$

and $\sigma=e^{\gamma}$ and $\gamma=$ Euler's constant $=.57721 \ldots \ldots$.

These results are the same as those obtained by Ritchie (1949), except that he has neglected terms with $k>1$. These Laplace inversion integrals were obtained rather ingeniously by him for specific values of $k$ and $z$. The present author has derived the general formula for the se integrals:

$$
I_{k, \nu}=\frac{(-1)^{k}(\tau)^{-(\nu+1)}}{\left[\log \left(\frac{4 \tau}{\sigma^{2}}\right)\right]^{k}} \sum_{s=1}^{N} \frac{(s+k-1) !}{s !(k-1) !} \frac{\psi^{(s)}}{\left(\log \frac{4 \tau}{\sigma^{2}}\right)^{s}}, \nu>-1
$$

where: $\quad \psi^{(s)}=\sum_{q=0}^{\frac{s-1}{2}, \frac{s-2}{2}}(-1)^{q} \pi^{2 q} \frac{s !}{(2 q+1) !(s-2 q-1) !} \frac{d^{s-2 q-1}}{d \nu^{5-2 q-1}} \Gamma(1+\nu)$

where the limit $(s-1) / 2$ is to be used when $s$ is odd, and $(s-2) / 2$ when $s$ is even.

and $(14)$

$$
I_{k,-\nu}=\frac{(-1)^{k}(\tau)^{\nu-1}}{\left[\log \left(\frac{4 \tau}{\sigma^{2}}\right)\right]^{k}} \sum_{s=0}^{N} \frac{(s+k-1) !}{s !(k-1) !} \frac{(-1)^{s}}{\left[\log \left(\frac{4 \tau}{\sigma^{2}}\right)^{s}\right.} \frac{d^{s}}{d \nu^{s}} \frac{1}{\Gamma(\nu)},-\nu<0
$$


The numerical coefficients of these series, for a few values of $k$ and $\nu$ are given by Ritchie (1049). It should be noted that the se series are asymptotic, and as such, the error cannot be made as small as one likes by taking $N$ very large but $\mathrm{N}$ must be so chosen that the error becomes minimum for a given value of $\mathcal{Z}$

At this point we shall discuss the concept of "radius of drainage"

(Muskat, 1937, p. 708). For large values of $\boldsymbol{Z}$ from equations (11) and (14)

$$
\begin{aligned}
\psi & \sim \Delta p^{2}\left[1+2 \log \rho I_{1,-1}(\tau)\right] \\
& \sim \Delta p^{2}\left[1-\frac{2 \log p}{\log \frac{4 \tau^{2}}{\sigma^{2}}}\right]
\end{aligned}
$$

which approximates the steady state form except that the radius of the reservoir changes with time. The steady state value is -

$$
\psi_{0}=\Delta p^{2}\left(1-\frac{\log \rho}{\log \frac{b}{a}}\right)
$$

Thus, we see that the pressure distribution approximates a steady state distribution when -

$$
\begin{aligned}
& \frac{2}{\log \frac{4 \tau}{\sigma^{2}}} \sim \frac{1}{\log \frac{b}{a}} \\
& \tau=\frac{\sigma^{2}}{4}\left(\frac{b}{a}\right)^{2}, \quad t=\frac{\mu F}{p_{e} k} \frac{\sigma^{2} b^{2}}{4} \sim 2 \times 10^{3} \mathrm{sec} .
\end{aligned}
$$

which agrees with Muskat's value numerically. However, the concept of "radius of drainage" is misleading, for the general behavior of the pressure distribution is given by equation (11). It should be noted that the velocity also becomes approximately the steady state velocity at the same value of $\boldsymbol{\mathcal { V }}$ as when the pressure reaches a steady state value, so that we can have a smooth joining of the solutions for the infinite and the finite reservoir problems.

We now consider the cumulative volume production per unit thickness, and find from equation (10) -

$$
\int_{0}^{t} Q d t \sim \frac{2 \pi a^{2}}{\kappa} \frac{k}{\mu} \frac{\Delta p^{2}}{p_{w}} I_{1,-2}
$$

$$
\text { where } \begin{aligned}
& I_{1,-2}(\tau)=-\frac{\tau}{y}\left(1+\frac{0.42218}{y}-\frac{0.46669}{y^{2}}-\frac{1.1465}{y^{3}}-\frac{0.58905}{y^{4}} \ldots\right) \\
& y=\log \frac{4 \tau}{\sigma^{2}}
\end{aligned}
$$


as given by Ritchie (1049). This approximation is good for -

$$
\tau>100 \text { or } t>0.1 \text { seconds. }
$$

It may be assumed that any contribution from $\tau<100$ may be ignored with the possible exception of $\boldsymbol{\tau}=\boldsymbol{O}$ where a step function discontinuity in the initial condition will introduce a singularity in the velocity and consequently the flux. However, the production is shown to be finite at $\boldsymbol{\tau}=0$ by evaluating equation (10) for large $z$

(19)

$$
\int_{0}^{t} Q d t \sim \tau^{1 / 2}
$$

For the sake of discussion of the errors involved in neglecting the nonlinearity of the equation, we list here the leading terms of the functions of interest to us.

$$
\begin{aligned}
p^{2} & =p_{e}^{2}-\psi=p_{e}^{2}-\Delta p^{2}\left[1+2 \log \rho I_{1,-1}(\tau)\right] \\
& \sim p_{e}^{2}-\Delta p^{2}\left[1-\frac{2 \log \rho}{\log \frac{4 \tau}{\sigma^{2}}}\left(1-\frac{0.57721}{\log \frac{4 \tau}{\sigma^{2}}}\right)\right]
\end{aligned}
$$

where

$$
\tau>\rho^{2}
$$

(21)

$$
\begin{aligned}
|\vec{v}| & \sim \frac{1}{p \rho} \frac{k}{\mu a} \Delta p^{2} I_{1,-1}(\tau) \\
& \sim \frac{-k}{\mu a} \frac{\Delta p^{2}}{p \rho} \frac{1}{\log \frac{4 \tau}{\sigma^{2}}}\left[1-\frac{0.57721}{\log \frac{4 \tau}{\sigma^{2}}}\right]
\end{aligned}
$$

$$
\begin{aligned}
\int_{0}^{t} Q d t & \sim \frac{-2 \pi a^{2}}{k} \frac{k}{\mu} \frac{\Delta p^{2}}{P_{w}}\left[-I_{1,-2}(\tau)\right] \\
& \sim \frac{-2 \pi k}{\mu} \frac{\Delta p^{2}}{P_{w}} \frac{t}{\log \frac{4 \tau}{\sigma^{2}}}\left(1+\frac{0.42278}{\log \frac{4 \tau}{\sigma^{2}}}+\cdots\right)
\end{aligned}
$$


As mentioned earlier, the only effect the nonlinearity has on the equation is in the change in effective "diffusivity", which is

$$
\frac{1}{\kappa \sqrt{1-\psi / p_{e}^{2}}}
$$

where the extreme values of $\psi$ are 0 and $\Delta p^{2}$. We have defined $K$ when $\psi=0$ by

$$
K=\frac{k}{\mu f} p_{e}
$$

When $\varphi$ takes on the other extreme value of $\Delta p^{2}$,

$$
K^{\prime}=\frac{K}{\mu f} P_{w}
$$

Therefore,

$$
\frac{K^{\prime}}{K}=\frac{P_{w}}{P_{e}}=\frac{a^{2} \tau^{\prime}}{a^{2} \tau}=\frac{\tau^{\prime}}{\tau}
$$

The diffusivity enter $s$ the above equations through $\boldsymbol{\tau}$, and the dominating term containing $\tau$ is $\log \frac{4 \tau}{\sigma^{2}}$ Thus, the maximum effect of variation in diffusivity is

$$
D=\frac{\frac{1}{\log \frac{4 \tau}{\sigma^{2}}}}{\frac{1}{\log \frac{4 \tau}{\sigma^{2}}}}=\frac{\log \frac{4 \tau}{\sigma^{2}}}{\log \frac{4 \tau}{\sigma^{2}}+\log \frac{p_{w}}{p^{e}}}
$$

If we take the hypothetical case of $P_{e}=100, P_{w}=75$ atmospheres, the diffusivity varies 25 percent. Then

$$
D \sim 1.03
$$

In other words, the fractional error in this ratio is 3 per cent at $\tau=10^{4}$ or $t=10$ sec. with correspondingly smaller errors for larger values of time. Thus, the effect of nonlinearity is small. 


\section{SUMMARY AND CONCLUSIONS}

We now summarize the result of this investigation. The expressions for the various quantities are -

$$
\text { (24a) } \begin{aligned}
p^{2}= & p_{e}^{2}-\Delta p^{2}\left\{\left\{1+2 \log \rho I_{1,-1}(\tau)\right\}+\right. \\
& +\frac{1}{4}\left\{2\left(\rho^{2}-1\right)(\log \rho-1) I_{1,0}(\tau)+4 \log \rho I_{2,0}(\tau)\right\}+ \\
& +\frac{1}{64}\left\{\left[2 \rho^{4}\left(\log \rho-\frac{3}{2}\right)-8 \rho^{2}(\log \rho-2)-13+6 \log \rho\right] I_{1,1}(\tau)+\right. \\
& +\left[16 \rho^{2}(\log \rho-1)-26 \log \rho+16\right] I_{2,1}(\tau)+ \\
& \left.\left.+32 \log \rho I_{3,1}(\tau)\right\}+\frac{2}{2304} O\left(\frac{\rho^{6}}{\tau^{3}}\right)\right\}
\end{aligned}
$$

when $\quad \rho^{2}<\tau \leqslant \frac{\sigma^{2}}{4}\left(\frac{b}{2}\right)^{2}$

(24b) $p^{2}=p_{e}^{2}-\Delta p^{2}\left(1-\frac{\log p}{\log \frac{b}{2}}\right)$

when

$$
\tau>\frac{\sigma^{2}}{4}\left(\frac{b}{2}\right)^{2}
$$

and 1

$$
\text { and (25a) } \begin{aligned}
|\vec{v}| & =\frac{-k}{2 \mu} \frac{\Delta p^{2}}{2 \rho P}\left\{-2 I_{1,-1}(\tau)+\frac{1}{4}\left\{\left[-2 \rho^{2}(2 \log \rho-1)+2\right] I_{1,0}(\tau)-\right.\right. \\
& \left.-4 I_{2,0}(\tau)\right\}+\frac{1}{64}\left\{\left[4 \rho^{4}\left(\frac{5}{2}-2 \log \rho\right)+8 \rho^{2}(2 \log \rho-3)-6\right] I_{1,1}(\tau)+\right. \\
& \left.+\left[26-16 \rho^{2}(2 \log \rho-1)\right] I_{2,1}(\tau)-32 I_{3,1}(\tau)\right\} \\
& \left.+\frac{6}{2304} O\left(\frac{\rho^{6}}{\tau^{3}}\right)\right\} \\
\text { when } \quad \rho^{2} & <\tau \leqslant \frac{\sigma^{2}}{4}\left(\frac{b}{a}\right)^{2}
\end{aligned}
$$

$$
|\vec{v}|=\frac{-k}{2 \mu} \frac{\Delta p^{2}}{\log \frac{b}{2}} \frac{1}{2 \rho} \frac{1}{\sqrt{p_{e}^{2}-\Delta p^{2}\left(1-\frac{\log \rho}{\log \frac{b}{2}}\right)}}
$$

when $\quad \tau>\frac{\sigma^{2}}{4}\left(\frac{b}{a}\right)^{2}$

by definition,

$$
Q=2 \pi a|\vec{v}|_{\rho=1}
$$




$$
\begin{aligned}
& \int_{0}^{t} Q d t=\frac{-2 \pi k}{\mu} \frac{\Delta p^{2}}{P_{w}} \frac{t}{y}\left(1+\frac{0.42278}{y}-\frac{0.46669}{y^{2}}-\right. \\
& \left.-\frac{1.1465}{y^{3}}-\frac{0.58905}{y^{4}}+\cdots\right)
\end{aligned}
$$

when $\quad 100<\tau \leqslant \frac{\sigma^{2}}{4}(b / a)^{2}$

where $\quad y=\log \frac{4 \tau}{\sigma^{2}}$ and $\quad \tau=\frac{p_{e} k t}{\mu f a^{2}}$

$$
\int_{t>\frac{\mu f}{p_{e} k}}^{t} \frac{\sigma^{2} b^{2}}{4}
$$

when

The equations denoted "a" refer to the transient state, and those denoted "b" to the steady state, as given by Muskat (1937).

The fractional error due to nonlinearity is -

$$
E=-\frac{\log \frac{P_{w}}{P_{e}}}{\log \frac{4 \tau}{\sigma^{2}}}
$$

which is shown to be small or negligible in most actual cases. It must be remembered that all these quantities are evaluated at well bottom pressure and temperature.

\section{ACKNOWLEDGMENTS}

The writer thanks R. H. Ritchie of the Oak Ridge National Laboratory for numerous discussions and clarification of obscure points.

\section{REFERENCES}

Carslaw, H. S., and Jaeger, J. C., Conduction of Heat in Solids, London, Oxford University Press, 1947.

Muskat, M., The Flow of Homogeneous Fluids Through Porous Media, New York, McGraw Hill, 1937.

Ritchie, R. H. , Some Problems in Conduction of Heat in a Cylindrical Coordinate System, Master's Thesis, University of Kentucky, 1949. 